\title{
RELIEVE CUMPLE 10 AÑOS, PERO... ¿CÓMO FUE EL PRIMERO?
}

\section{(RELIEVE turns 10 years, but... how it was the first?)}

\author{
por
}

Article record

About authors

Gregorio Rodríguez Gómez (gregorio.rodriguez@uca.es)

$\underline{\text { Ficha del artículo }}$

$\underline{\text { HTML format }}$

$\underline{\text { Sobre los autores }}$

Formato HTML

\begin{tabular}{|c|}
\hline $\begin{array}{l}\qquad \text { Abstract } \\
\text { The author remembers the beginnings of RELIEVE, the } \\
\text { first electronic journal that was authorized in Spain. The } \\
\text { evolution of electronic publishings in these ten years is } \\
\text { also analyzed. }\end{array}$ \\
\hline Keywords \\
\hline $\begin{array}{l}\text { Scholarly Journals; Electronic Journals; Electronic Pub- } \\
\text { lishing; Information Dissemination; Internet }\end{array}$ \\
\hline
\end{tabular}

\section{Introducción}

Dicen que cualquier innovación supone un esfuerzo y, sobre todo, una lucha contra quienes desean permanecer sin cambiar. El caso de las publicaciones electrónicas no iba a ser menos.

La actual dirección de RELIEVE me sugirió hace tiempo que, con motivo del décimo aniversario del inicio de la publicación, realizara una breve semblanza de los momentos iniciales en los que tuve el reto y el honor de iniciar esta publicación. Tras muchas insistencias por parte del actual equipo de dirección y numerosas respuestas por mi parte del tipo “a ver si lo hago”, “estoy en ello” o “interesante...", siempre denotando una cierta resistencia, motivada quizás por la desazón que puede dar la nostalgia, me encuentro ahora intentando cumplir con ese "encargo".

Cuando tuve la idea de abordar la creación desde nuestra Asociación Interuniversitaria de Investigación Pedagógica (AIDIPE) de una revista que tuviera formato electrónico, fue muy bien acogida por parte del por entonces presidente (Javier Tejedor) quien ejerció, desde el primer momento, no sólo como primer director de la revista sino, lo más importante, como impulsor de la misma; pero fueron muchos los retos que tuvimos que ir abordando para que la revista saliera a la red. De ellos 
paso a evocar los que ahora, con el paso del tiempo, recuerdo con más emotividad.

\section{El reto de las infraestructuras}

El primer obstáculo que había que enfrentar era el de disponer de las infraestructuras mínimas necesarias que pasaban por disponer de una conexión a Internet y un servidor donde almacenar la información.

En aquel momento (1993/94) prestaba mis servicios como Profesor Ayudante en la Universidad de Sevilla, y éramos realmente pocos los que teníamos acceso (y conocimiento, todo hay que decirlo) simplemente al correo electrónico; tan es así que la conexión a Internet tuvimos que "robarla", de forma que utilizando un cable que atravesaba los tejados de la Facultad conseguimos unir uno de los ordenadores del despacho con los ordenadores del centro de cálculo.

Ahora todos (o casi todos) disponemos de $\mathrm{Wi}-\mathrm{Fi}$ en nuestros centros y es común ver a profesorado y alumnado conectándose a Internet a través de la red inalámbrica; pero en aquel momento de lo que disponíamos más bien era de una red de profesores en torno a un solo ordenador conectado a través de un cable que recorría serpenteante por el despacho, salvando sillas y mesas, y que era preciso sortear para no tropezar con él; hecho éste último que sucedía con más frecuencia de la debida con la consiguiente desconexión a la red y la necesaria atención de primeros auxilios al compañero que acababa de ser literalmente “atrapado por la red".

Menos difícil fue disponer del servidor donde almacenar los números de la revista, que fue facilitado por el Centro de Informática Científica de Andalucía (CICA) que en todo momento puso a nuestra disposición no sólo sus medios sino también su saber hacer.

Así que pertrechado con conexión y servidor, ahora se trataba de trasladar la información de un lado a otro. No os vayáis a pensar que se podía hacer como ahora que enviamos de todo por la red. En ese momento tan sólo podíamos enviarnos ficheros en formato texto para no tener problemas de compatibilidad. Y como el correo electrónico a veces era inestable, pues nada, a pasear con el disco flexible de 5,25" desde la Facultad hasta el CICA, y así fuimos montando lo que sería la base de RELIEVE: Portada, normas, miembros del Consejo de Redacción ... etc.

\section{El reto del ISSN}

El primer paso para abordar el reconocimiento de una publicación es solicitar el ISSN (International Standard Serial Number / Número Internacional Normalizado de Publicaciones Seriadas) que es el código internacional de identificación de las publicaciones seriadas (revistas, periódicos, boletines, anuarios, series de monografías...).

Actualmente ya podemos solicitar este número, incluso para las revistas electrónicas, a través de la página Web de la Biblioteca Nacional pero, por aquel entonces, tuvimos que enviar una carta explicando con todo lujo de detalles lo que nos traíamos entre manos. Os podréis imaginar la respuesta inmediata: "Eso no puede ser. Una revista tiene que ser impresa”.

Así que tuvimos que proceder a "convencer" a la responsable de entonces de lo que era una revista electrónica, aportando como claro ejemplo de ello la "Educational Policy Analysis Archives" que siempre ha sido un referente en este sentido. Para ello tuvimos que enviar los fichero originales, grabados en disco flexible, de todo lo que aparecía en la red acompañado de su correspondiente copia impresa, así como un memorandum explicativo de lo que eran las revistas electrónicas.

No os podéis ni imaginar la alegría que nos produjo el recibir esa carta del Ministerio de Cultura, y escrito "a máquina”, en la que se nos comunicaba oficialmente el número ISSN de nuestra revista. $\mathrm{Y}$ a fin de compartir esa alegría y que quede constancia para un futuro digital, lo adjuntamos en el anexo I. Además, aprovecho para agradecer así públicamente el buen hacer de la entonces Jefe de la Sección del ISSN D ${ }^{\mathrm{a}}$ Lucía Sagredo Miller. 
Posteriormente, con mi incorporación como profesor a la Universidad de Cádiz (UCA) y por aquello de tenerla "cerca”, procedimos en 1997 al cambio de ubicación de la revista a los servidores de la UCA. Este cambio tuvimos que comunicarlo al Ministerio de Cultura, lo cual nos agradeció $\mathrm{D}^{\mathrm{a}}$ Lucía Sagredo al tiempo que nos recomendaba que le comunicáramos cualquier otro cambio que se produjera en cuanto a periodicidad, localización, etc., como podemos ver en el fax recogido en el anexo II.

\section{El reto de los consejeros}

Establecer un primer consejo de redacción no fue tarea fácil ya que, además de los tradicionales criterios de competencia y reconocimiento científico, en este caso los miembros del consejo debían disponer de correo electrónico y unos conocimientos mínimos para utilizar los recursos de la red. Así pues, resultó algo complicado y difícil encontrar personas de nuestro entorno educativo que dispusiera de esos medios.

No obstante, recuerdo en estos momentos con enorme satisfacción y alegría aquellos correos electrónicos en "fósforo verde" que nos enviaban desde el otro lado del atlántico, sobre todo y de manera muy especial la aceptación como miembro del consejo asesor por parte de Robert Stake o el de Margaret D. LeCompte aceptando la publicación en castellano de su trabajo presentado previamente en la reunión de la AERA y que constituyó el $n^{0} 1$ de la revista.

\section{El reto de sacarla a la red}

En la actualidad nos movemos por la red con nuestro navegador (Explorer, Netscape, Gozzilla... etc.) y todos participan de elementos comunes, pero en aquel momento inicial teníamos que partir de una herramienta básica de la que muchos ni conoceréis el nombre. Por cierto que hasta a mí se me ha olvidado en el momento de escribir estas páginas $\mathrm{y}$ he tenido que recurrir a mi archivo documental para recordarlo: "Gopher” (Rodríguez y otros: 1995). Con este navegador se podía organizar la información de forma jerárquica arbórea. Nos podemos hacer una leve idea de la "riqueza gráfica y visual” al observar la figura 1 donde, además, podemos comprobar la fecha de envío del primer número de RELIEVE: sábado 14 de Enero de 1995.

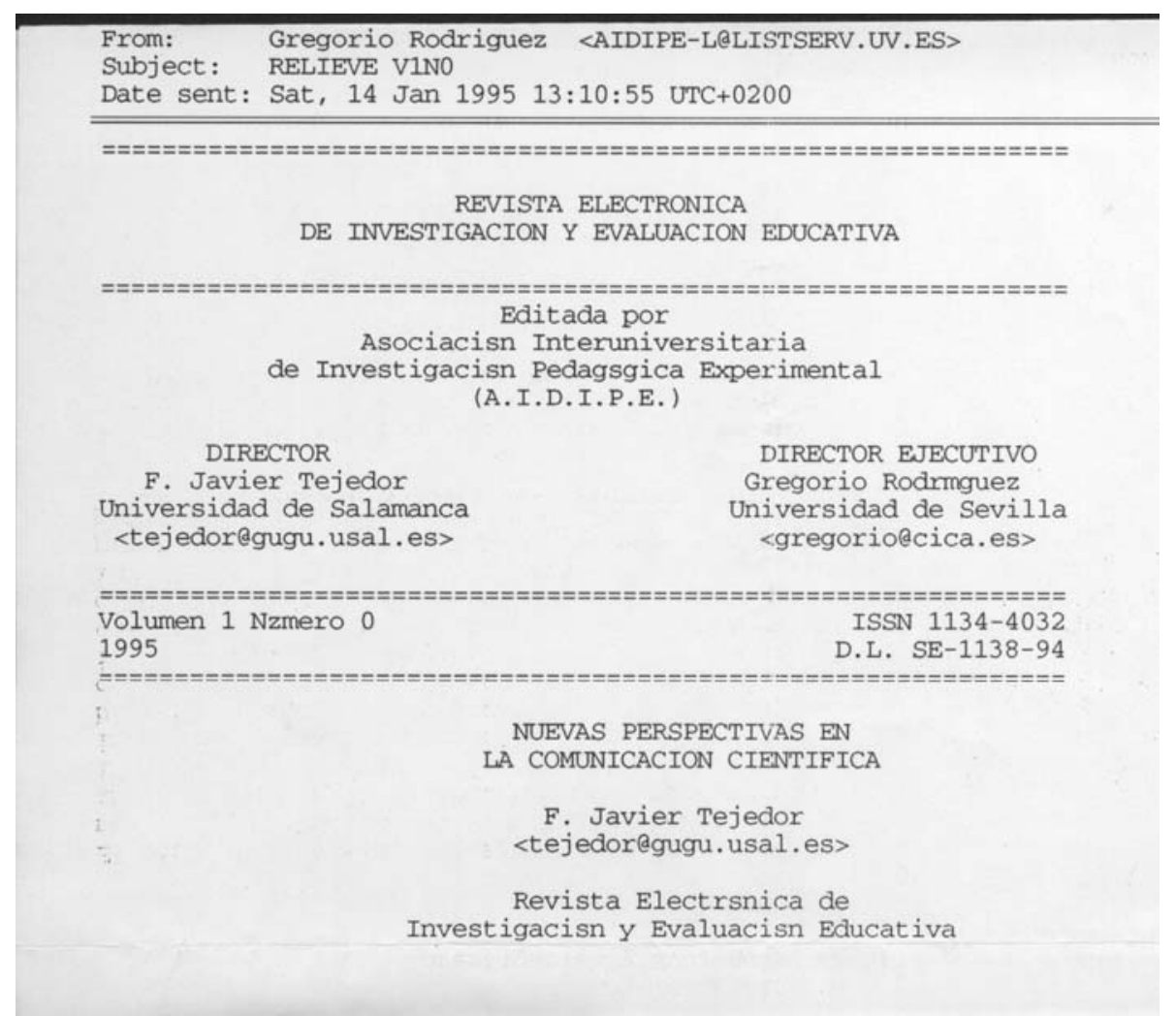

Figura 1: Primer artículo de RELIEVE 
Rodríguez Gomez, G. (2005). RELIEVE cumple 10 años, pero... ¿Cómo fue el primero?. RELIEVE: v. 11, n. 2, p. 103-112. http://www.uv.es/RELIEVE/v11n2/RELIEVEv11n2_1.htm

O los menús iniciales que aparecían en cuando se consultaba la revista servidores
Gopher como los que se presentan en la Figuras 2 y $3^{[i]}$.

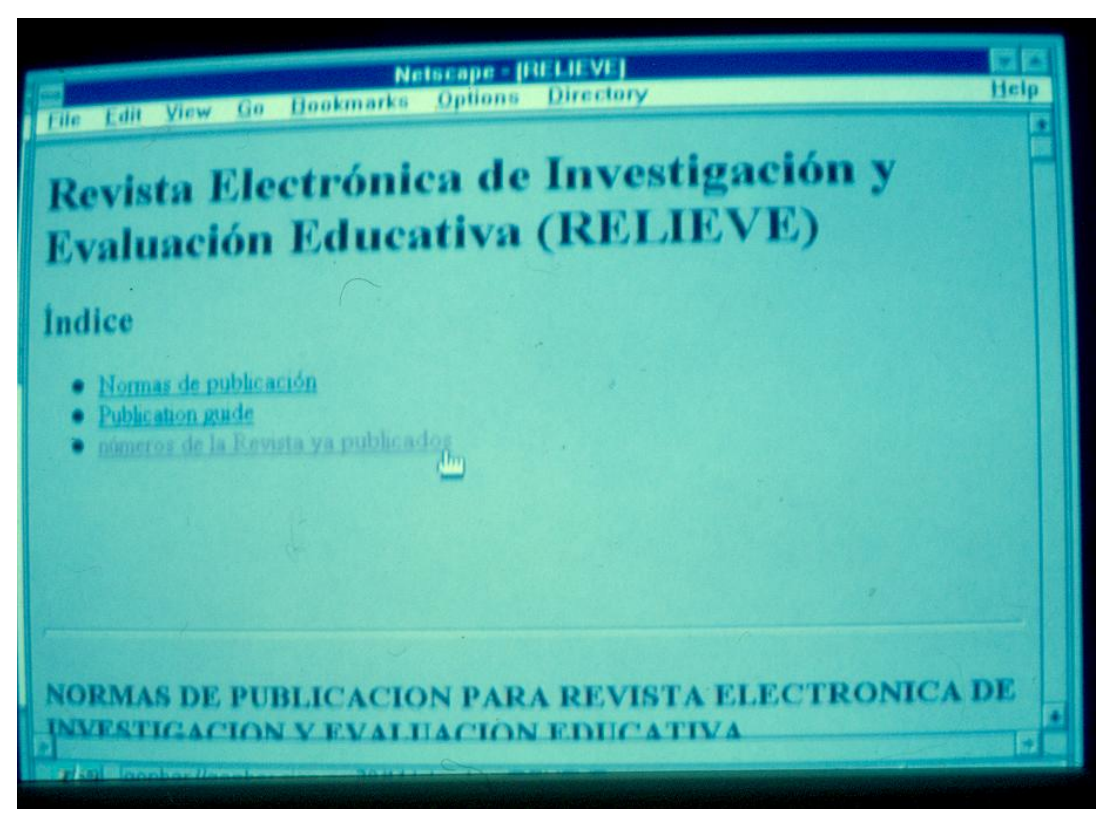

Figura 2: Menús iniciales de RELIEVE a través del servidor Gopher (I).

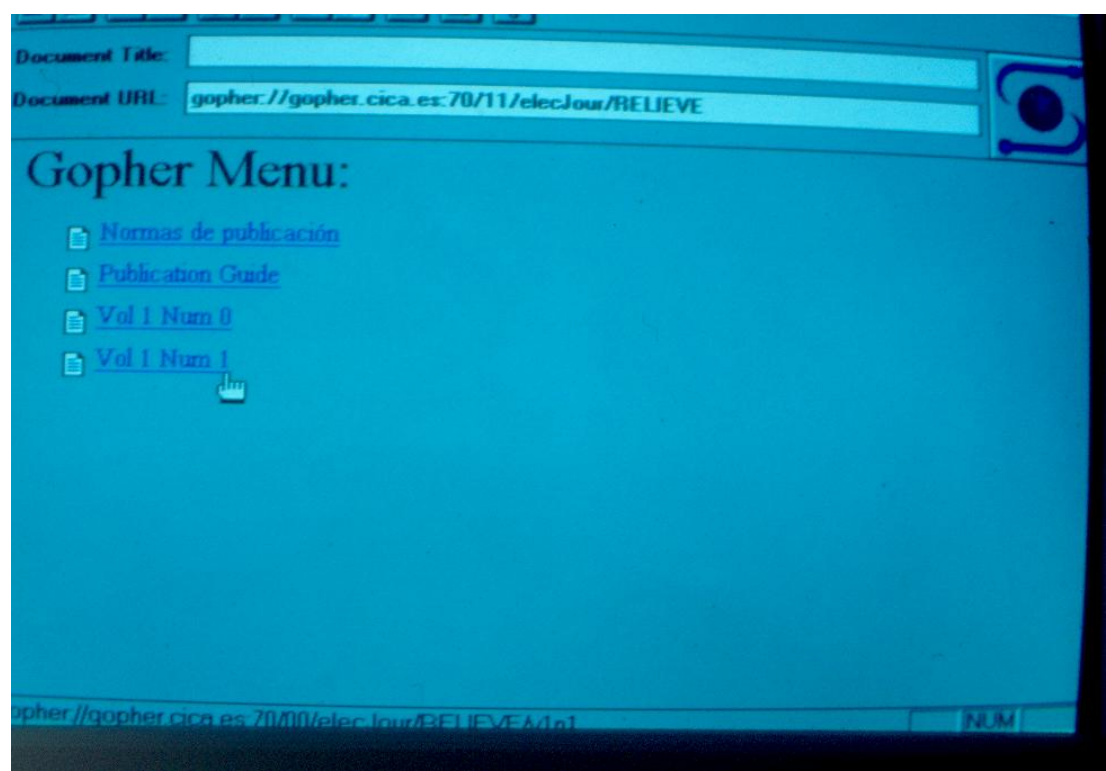

Figura 3: Menús iniciales de RELIEVE a través del servidor Gopher (II).

Posteriormente se aprovechó el cambio a la UCA para modificar también la forma de acceso. Así pasamos del Servidor Gopher del CICA al servidor World Wide Web (WWW) de la UCA. Fue el salto al formato HTML.
Ya se disponía de los primeros navegadores, por lo que la portada se cambió al formato que se presenta en la figura 4 introduciendo colores y las nuevas posibilidades que daba el naciente formato. 


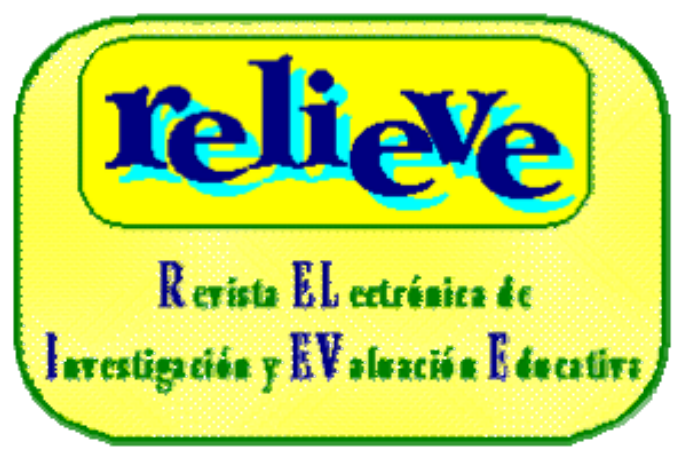

Director:

F. Javior Tejedor

universbiad de Salamanod

tejador grupususales

Director ejeoutivo:

Gregerto godrigtes

anderidad do chils

gogorio rodrizuastueses

\section{Una publecacion electrónicar editada por la

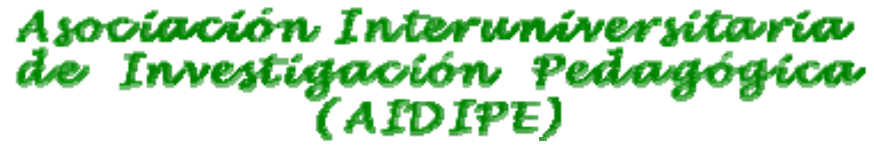

Figura 4: Página de presentación de RELIEVE en 1997

\section{El reto de la presentación en público}

En Septiembre de 1995 celebramos el VII Seminario de Modelos de Investigación Educativa en Valencia y, en ese contexto, tuvimos la oportunidad de compartir una mesa redonda en la que me tocó presentar las revistas electrónicas como una nueva posibilidad de comunicación en la investigación educativa (Tejedor y otros, 1995). Fue el momento de presentación en público de RELIEVE y para ello editamos un díptico donde se explicaba la forma de suscripción y acceso a los números de la revista (Anexo III).

La repercusión de la revista fue rápida, y ya en 1996 se citaba a RELIEVE como una "revista abierta a todos” (Aliaga, 1996). De hecho, en los nueve primeros meses de funcionamiento de RELIEVE se produjeron un total de 1.613 accesos al directorio de la misma y por entonces mantuve que "a través de estos datos podemos comprobar el elevado potencial de comunicación que supone una revista de estas características” (Tejedor y otros, 1995). En la figura 5 podemos observar la secuencia de visitas a la revista en los últimos meses y en la tabla 1 la distribución del número de visitas por países de origen. Podemos comprobar así el aumento exponencial que se ha producido desde entonces hasta ahora y el número de países a los que se llega indicando así su influencia a nivel internacional.

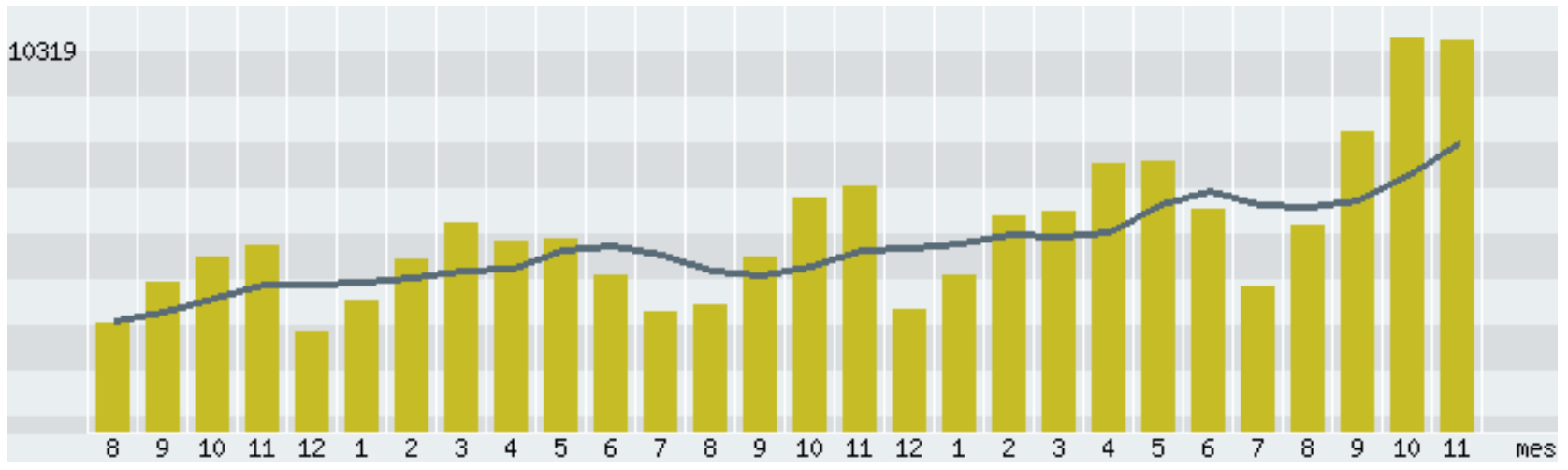

Figura 6: Visitas a RELIEVE por mes. Datos de Noviembre de 2005

(Fuente http://www.webstats4u.com) 
Rodríguez Gomez, G. (2005). RELIEVE cumple 10 años, pero... ¿Cómo fue el primero?. RELIEVE: v. 11, n. 2, p. 103-112. http://www.uv.es/RELIEVE/v11n2/RELIEVEv11n2_1.htm

\begin{tabular}{|c|c|c|c|}
\hline 1. & México & 37882 & $22.7 \%$ \\
\hline 2. & España & 36756 & $22.0 \%$ \\
\hline 3. & Venezuela & 14804 & $8.9 \%$ \\
\hline 4. & Argentina & 11157 & $6.7 \%$ \\
\hline 5. & Chile & 10065 & $6.0 \%$ \\
\hline 6. & Estados Unidos & 10057 & $6.0 \%$ \\
\hline 7. & Colombia & 9301 & $5.6 \%$ \\
\hline 8. & Perú & 7929 & $4.8 \%$ \\
\hline 9. & Puerto Rico & 6023 & $3.6 \%$ \\
\hline 10. & Uruguay & 5137 & $3.1 \%$ \\
\hline 11. & Bolivia & 1850 & $1.1 \%$ \\
\hline 12. & República Dominicana & 1506 & $0.9 \%$ \\
\hline 13. & Costa Rica & 1127 & $0.7 \%$ \\
\hline 14. & El Salvador & 939 & $0.6 \%$ \\
\hline 15. & Panamá & 938 & $0.6 \%$ \\
\hline 16. & Ecuador & 888 & $0.5 \%$ \\
\hline 17. & Guatemala & 884 & $0.5 \%$ \\
\hline 18. & Brasil & 794 & $0.5 \%$ \\
\hline 19. & Portugal & 791 & $0.5 \%$ \\
\hline 20. & Cuba & 554 & $0.3 \%$ \\
\hline 21. & Reino Unido & 284 & $0.2 \%$ \\
\hline 22. & Alemania & 260 & $0.2 \%$ \\
\hline 23. & Canadá & 202 & $0.1 \%$ \\
\hline 24. & Australia & 183 & $0.1 \%$ \\
\hline \multirow[t]{4}{*}{25.} & Nicaragua & 168 & $0.1 \%$ \\
\hline & Desconocido & 4791 & $2.9 \%$ \\
\hline & El resto & 1655 & $1.0 \%$ \\
\hline & Total & 166925 & $100.0 \%$ \\
\hline
\end{tabular}

Terminaba mi participación en aquella mesa redonda afirmando: "en la medida en que se vaya produciendo una mayor participación por parte de los investigadores no cabe duda que esto que hoy puede considerarse, por parte de algunos, como exótico, en un plazo no demasiado lejano será "una realidad virtual real", alternativo y/o complementaria a otros medios de relación y comunicación entre investigadores basado, hasta ahora, en el encuentro cara a cara (caso de los congresos y jornadas, por ejemplo) y el formato de papel (caso de las revistas tradicionales)" (Tejedor y otros, 1995).

Han pasado ya 10 años y RELIEVE se ha convertido en un referente como publicación electrónica en nuestro país, sobre todo gracias al enorme entusiasmo y esfuerzo realizado por parte del actual equipo directivo de la misma. Mi gratitud personal a ellos por asumir el reto de darle continuidad a una idea, y mi gratitud profesional por- 
que gracias a ellos disponemos desde AIDIPE de un medio de comunicación y divulgación científica reconocido a nivel nacional e internacional y han sido capaces de conseguir "una realidad virtual real”.

La aportación a la mesa redonda la cerraba con una cita de Lincoln y Denzin (1994) de acuerdo con la cual el futuro que se nos avecinaba se basaría "en la interacción ciberespacial, alojada y creada en la realidad virtual del texto electrónico", y en este momento la parafrasearía así:

\section{ver vídeo}

"El futuro que se nos avecina se basará en la interacción ciberespacial, alojada y creada en la realidad digital. Dentro de 10 años... más”

\section{Referencias}

Aliaga F. (1996). Enredados: aplicaciones y experiencias de Internet en España con interés educativo. Bordón, 48 (3), 355-361.

Lincoln, Y.G. y Denzin, N.K. (1994). The Fith Moment. En N.K. Denzin \& Y.S. Lincoln (Eds.). Handbook of Qualitative Research, pp. 575-586. Londres: Sage.

Rodríguez, G. (1999). Revistas electrónicas: Cibernautas y/o papirófilos. Cambios en la comunicación científica. Revistas de Investigación Educativa, 17 (2), 491-494.

Rodríguez, G. y otros (1995). El tratamiento de la información en la investigación educativa (Una propuesta informatizada en entorno PC). PixelBit. Revista de Medios y Educación, (5), 55-73.

Tejedor, F.J., Aliaga, F., González Such, E., López González, E., Rodríguez Gómez, G y Salvador, L. (1995). Las redes de información. Revista de Investigación Educativa, (26), 287-314.

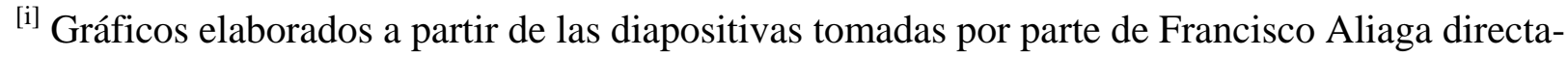
mente sobre la pantalla del ordenador cuando se inició la publicación de RELIEVE.

\section{AGRADECIMIENTOS}

Mi agradecimiento a Francisco Aliaga, quien insistentemente me empujó a realizar esta añoranza y me ayudó a rescatar imágenes y papeles del recuerdo para que pasen a convertirse en fondo "digital”. 
Rodríguez Gomez, G. (2005). RELIEVE cumple 10 años, pero... ¿Cómo fue el primero?. RELIEVE: v. 11, n. 2, p. 103-112. http://www.uv.es/RELIEVE/v11n2/RELIEVEv11n2_1.htm

\section{Anexo I:}

FACILTRD DE FILOSOEIA Y CIENICIAS DE LA EDJCACTCN

Asociaclán Interuntversitarla de Invest'gación

Pedagóof Câ Experimental

Auda. San Francisco Javier, s/n

4) 1 WDS SEVILLA.

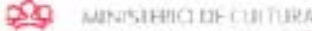 B) Biblioteca NaCionai.}

CENTRO NACIONAL FSPANOL IS8N

Madrid, 20 de Julio de 1.994

Señores:

El Centro Nacional Español ISSN asigna un Numero Internacional Normalizado de Publieaciones en Serie ISSN (Internнeional Standard Serial Number) a las publicaciones en serie editadas en España, es decir, a las revistas, periódicos, boletines, publicnciones anuales, elc.

Se recomienda que los editares e impresores impriman el ISSN en cada fascien lo de la publicación correspondiente, en la parte alta y a la derecha de la cubierta o jun to al numero de Depósi to Legal a del ISBN, si tiece (ISSN e ISIN nu sun códigos incompatibles). Se escribe en dos grupos de cuatro cifras separadas por un guión y precedido siempre de las siglas ISSN, por ejemplo: ISSN 1234-5678 (el úl timo dígito podrá ser una $X$ ).

Este número constituye una referencia sencilla y univoca de identificación de la publicación correspondiente, que facilita su control por parte de todos los agentes implicados, editores, distribuidores y bibliotecas, permite una comunicación más eficiente entre los mismos y está ideado para el Lratamienta a utomatizadn.

ATKNCION: el ISSN esté indisolublemente unido al titula normalizado, o título clave, de la publicación por lo que cada cambio de titulo supone la asignacioin de un nuevo ISSN. Por tanto, si se produce un cambio de titulo, por superficial que les parezca (incluso si se trata de un cambio en el orden y disposición de las palabras', o si se cambia el nombre de la institución eritora, tendrán que ponerse en contacto con el Centro Nacional Español del ISSN para que éste decida si corresponde la asignación de un nuevo ISSN.

Los elementos de identificación de la publicación editada por ustedes, son los siguientes:

ISSN $\quad 1734-4032$

Titulo clave: Revista electrónica de investigación y evaluación Titulo abreviaducativa

Título abreviado: Rev. electron. investig. eval. educ.

Les saluda atentamente,

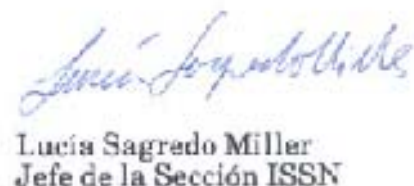

Jefe de la Sección ISSN

Telefonos decontacto: (91) 5807843 y $530 ; 728$

Escrito de comunicación del ISSN (106 Kb) 
Rodríguez Gomez, G. (2005). RELIEVE cumple 10 años, pero... ¿Cómo fue el primero?. RELIEVE: v. 11, n. 2, p. 103-112. http://www.uv.es/RELIEVE/v11n2/RELIEVEv11n2_1.htm

\section{Anexo II:}

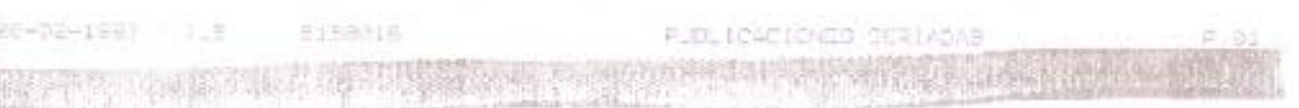

Anexo III: Díptico de presentación de RELIEVE (1995) (pdf- $470 \mathrm{~Kb}$ ) 
Rodríguez Gomez, G. (2005). RELIEVE cumple 10 años, pero... ¿Cómo fue el primero?. RELIEVE: v. 11, n. 2, p. 103-112. http://www.uv.es/RELIEVE/v11n2/RELIEVEv11n2_1.htm

\section{ABOUT THE AUTHORS / SOBRE LOS AUTORES}

Gregorio Rodríguez Gómez (gregorio.rodriguez@uca.es). Profesor Titular del Área de Métodos de Investigación y Diagnóstico en Educación de la Universidad de Cádiz. Fue el fundador de la RELIEVE y su director ejecutivo desde 1994 hasta el año 2001. Su dirección postal es: Facultad de Ciencias de la Educación. Area MIDE. Campus Río San Pedro. 11519 - PUERTO REAL (Cádiz).

\section{ARTICLE RECORD / FICHA DEL ARTÍCULO}

\begin{tabular}{|c|c|}
\hline $\begin{array}{l}\text { Reference / } \\
\text { Referencia }\end{array}$ & $\begin{array}{l}\text { Rodríguez Gómez, Gregorio (2005). RELIEVE cumple } 10 \text { años, pero … ¿cómo fue el } \\
\text { primero?. Revista ELectrónica de Investigación y EValuación Educativa, v. 11, n. } 2 \text {. } \\
\text { http://www.uv.es/RELIEVE/v11n2/RELIEVEv11n2_1.htm Consultado en (poner fe- } \\
\text { cha). }\end{array}$ \\
\hline Title / Título & $\begin{array}{l}\text { RELIEVE cumple } 10 \text { años, pero ... ¿cómo fue el primero?. [RELIEVE turns } 10 \text { years, } \\
\text { but... how it was the first?] }\end{array}$ \\
\hline Authors / Autores & Gregorio Rodríguez Gómez \\
\hline $\begin{array}{l}\text { Review / } \\
\text { Revista }\end{array}$ & Revista ELectrónica de Investigación y EValuación Educativa (RELIEVE), v. 11, n. 2 \\
\hline ISSN & $1134-4032$ \\
\hline $\begin{array}{l}\text { Publication date / } \\
\text { Fecha de publicación }\end{array}$ & $\begin{array}{l}2005 \text { (Reception Date: } 2005 \text { December 05; Approval Date: } 2005 \text { December 29; Pub- } \\
\text { lication Date: } 2005 \text { December 30) }\end{array}$ \\
\hline $\begin{array}{l}\text { Abstract / } \\
\text { Resumen }\end{array}$ & $\begin{array}{l}\text { The author remembers the beginnings of RELIEVE, the first electronic journal that was } \\
\text { authorized in Spain. The evolution of electronic publishings in these ten years is also } \\
\text { analyzed.. } \\
\text { El autor recuerda los inicios de RELIEVE, la primera revista electrónica que se autori- } \\
\text { zó en España. Se analiza también la evolución de las publicaciones electrónicas en estos } \\
\text { diez años }\end{array}$ \\
\hline $\begin{array}{l}\text { Keywords } \\
\text { Descriptores }\end{array}$ & $\begin{array}{l}\text { Scholarly Journals; Electronic Journals; Electronic Publishing; Information Dissemina- } \\
\text { tion; Internet. } \\
\text { Revistas académicas; Revistas Electrónicas; Edición Electrónica; Comunicación de la } \\
\text { Información; Internet; }\end{array}$ \\
\hline $\begin{array}{l}\text { Institution / } \\
\text { Institución }\end{array}$ & Universidad de Cádiz (Spain) \\
\hline $\begin{array}{l}\text { Publication site / } \\
\text { Dirección }\end{array}$ & http://www.uv.es/RELIEVE \\
\hline Language / Idioma & Español (Title, abstract and keywords in english ) \\
\hline
\end{tabular}

Revista ELectrónica de Investigación y EValuación Educativa (RELIEVE)

[ ISSN: 1134-4032 ]

(C) Copyright, RELIEVE. Reproduction and distribution of this articles it is authorized if the content is no modified and their origin is indicated (RELIEVE Journal, volume, number and electronic address of the document).

(C) Copyright, RELIEVE. Se autoriza la reproducción y distribución de este artículo siempre que no se modifique el contenido y se indique su origen (RELIEVE, volumen, número y dirección electrónica del documento). 